\title{
An observational efficacy and safety analysis of the treatment of acute invasive aspergillosis using voriconazole
}

\author{
F. Jacobs • D. Selleslag $\cdot$ M. Aoun • A. Sonet $•$ \\ A. Gadisseur
}

Received: 31 March 2011 / Accepted: 12 September 2011/Published online: 5 October 2011

(C) The Author(s) 2011. This article is published with open access at Springerlink.com

\begin{abstract}
The purpose of this study was to evaluate efficacy and safety of voriconazole in patients with acute invasive aspergillosis (IA) in a real-life, clinical setting. This was a multicenter observational study in adult patients treated with voriconazole for invasive mycosis. The study evaluated clinical response, mortality, use of other licensed antifungal therapy (OLAT), and treatment duration. This sub-analysis evaluated treatment and outcome data specifically from adult patients with proven/probable IA, while safety data were assessed in patients with proven/probable/ possible IA. Of the 141 patients enrolled, 113 were adults with proven/probable IA and six had possible IA. Voriconazole treatment duration ranged from 1 to 183 days (median, 49.5 days). Voriconazole was used exclusively in $64 \%(72 / 113)$ of patients and in combination/sequentially with OLAT in $36 \%$. Overall successful treatment response was $50 \%$ (57/113 patients). Twelve percent (14/113) of
\end{abstract}

F. Jacobs $(\bowtie)$

Hôpital Erasme, Université Libre de Bruxelles (ULB),

Brussels, Belgium

e-mail: frederique.jacobs@erasme.ulb.ac.be

D. Selleslag

AZ Sint-Jan Hospital,

Brugge, Belgium

M. Aoun

Institut Bordet,

Brussels, Belgium

\section{A. Sonet}

Hôpital Universitaire de Mont-Godinne,

Yvoir, Belgium

A. Gadisseur

Antwerp University Hospital,

Edegem, Belgium patients were switched to OLAT, either because of insufficient response (four patients) or for safety reasons (10 patients). Overall and attributable (entirely or partially due to fungal infection) mortality rates were 52\% (59/113) and $17 \%$, respectively. Treatment-related adverse events were reported for $18 \%(22 / 119)$ of patients. This observational study confirms the results of previous clinical trials demonstrating voriconazole as an effective and safe agent for treatment of confirmed acute IA.

\section{Introduction}

The incidence of severe systemic fungal infections has increased over the past decade [1, 2], mainly due to the increasing number of immunosuppressed patients surviving with underlying disease (e.g., hematologic/non-hematologic malignancies, HIV) and those undergoing hematopoietic stem cell or solid organ transplantation [2-4]. Such infections represent a serious cause of morbidity and mortality in these patient populations $[1,5,6]$.

Voriconazole is a broad-spectrum triazole antifungal agent approved in the European Union and the United States for the treatment of invasive aspergillosis (IA), candidemia in non-neutropenic patients, specific types of invasive Candida infections, and serious fungal infections caused by Scedosporium and Fusarium spp. [7, 8]. Of note, this agent is not active against the zygomycetes [9]. The efficacy of voriconazole was demonstrated in several clinical studies [10-12] and in a randomized, open-label, comparative trial of 277 patients with acute IA [13]. In that trial, voriconazole showed superior efficacy and tolerance compared with conventional amphotericin B in all patient populations [13]. Voriconazole is now generally recommended as first-line therapy of IA [14-17], and in Belgium 
it is reimbursed for the treatment of proven or probable IA (according to international consensus criteria), among other conditions.

While voriconazole has demonstrated efficacy in clinical trials, data from observational studies prospectively conducted in a real-life setting are sparse. Observational evidence may add relevant information to the totality of clinical evidence [18], and can thus be regarded as complementary to randomized clinical trials. Such real-life data are important not only to clinicians faced with selecting appropriate antifungal treatment, but also to healthcare payers making reimbursement decisions. For instance, observational studies can help to assess the predictive and external validity of pharmacoeconomic models, such as the model used to support the reimbursement submission of voriconazole in Belgium [19]. We therefore conducted a study to evaluate the use of voriconazole in daily clinical practice for the treatment of invasive mycoses in Belgian hospitals, with a specific focus on adult patients with acute IA. Treatment and outcome data were evaluated in patients with proven/probable IA, while safety was assessed in patients with proven/probable/ possible IA.

\section{Materials and methods}

Study design and population

This was a prospective, multicenter, observational, noninterventional study in adult patients treated for serious invasive fungal infections. The study was conducted in various clinical-care settings (hematology, infectious disease, pulmonary medicine, and intensive care) in Belgian hospitals. Ten centers (mainly major academic hospitals), which routinely treat patients with IA, initially participated in this study.

Each participating investigational site was asked to collect data from 15 to 20 successive eligible patients over a period of approximately 18 months. Patients were eligible for inclusion into the study if they received intravenous (IV) or oral voriconazole for first-line treatment of acute invasive aspergillosis, candidiasis, or scedosporiosis. Of note, the type of antifungal therapy (including dosing and duration) was selected entirely at the local investigator's discretion. Diagnosis and classification (i.e., as proven, probable, or possible) of invasive mycoses was carried out by the investigator according to generally accepted standard criteria developed by the European Organization for Research and Treatment of Cancer (EORTC) together with the National Institute of Allergy and Infectious Diseases Mycosis Study Group (MSG) [20], and was based on a combination of histologic, microbiologic, and radiologic evidence.
Each site acquired Institutional Review Board/Independent Ethics Committee approval of the study design. The study was performed in accordance with the ethical standards laid down in the Declaration of Helsinki. All patients gave their written informed consent. In seriously ill patients who were unable to make properly informed decisions, consent was obtained from the next of kin or legal representative.

\section{Treatment and outcome evaluations}

Data collection started on day 1 of voriconazole therapy and continued for a maximum duration of 182 days. Patients were followed until the end of antifungal therapy or day 182; treatment durations of more than 182 days were recorded as 183 days. The primary clinical endpoint was the patient's response to antifungal therapy, assessed either at 12 weeks of treatment or at the end of therapy. Outcomes were categorized by the investigator as complete response (i.e., resolution of all clinical signs and symptoms and most of the radiologic lesions), partial response (i.e., major improvement), stable (i.e., minor or no improvement, but no deterioration), or failure, based on the change in status of symptoms, signs, and/or diagnostic abnormalities attributable to invasive mycosis that had been present at baseline. Failure was defined as deterioration in attributable symptoms, signs, and/or diagnostic abnormalities present at baseline. Complete or partial clinical responses, but not stable disease, were considered successful treatment responses. Use of other licensed antifungal therapy (OLAT) was not considered a failure if the patient had a successful response at 12 weeks. These definitions of outcomes and treatment response are identical to those used in the pivotal randomized clinical trial of voriconazole in IA [13]. Other endpoints were survival at the end of the observational period, number of patients who also received OLAT, and treatment duration. In terms of treatment and outcome evaluations, this sub-analysis focuses exclusively on adult patients with proven/probable, acute IA. Patients aged $<18$ years who received oral voriconazole $200 \mathrm{mg}$ twice daily (i.e., the same dosage recommended for adults) [7, 8] were classified as adults for the purposes of this study and were consequently included in these analyses; patients aged $<18$ years who received $<200 \mathrm{mg}$ oral voriconazole twice daily were excluded.

\section{Safety evaluations}

Adverse events (AEs) were monitored throughout the study across all enrolled patients and were reported spontaneously by the physician. Laboratory values of aspartate aminotransferase, alanine aminotransferase, bilirubin, alkaline phosphatase, and serum creatinine were considered abnor- 
mal when they exceeded five times the upper limit of normal (or $2.5 \mathrm{mg} / \mathrm{dl}$ for serum creatinine). Treatmentemergent laboratory abnormalities were defined as those that worsened (i.e., new abnormality or change in severity) during treatment compared with baseline levels. In terms of safety evaluations, this paper focuses exclusively on adult (according to the definition above) patients with proven/ probable/possible acute IA.

\section{Statistical methods and sample size}

The study was designed to prospectively collect data from 120 to 150 eligible patients. All analyses were based on the intent-to-treat population, defined as those patients who received at least one dose of study medication. Descriptive statistics were used to explore the collected data.

\section{Results}

\section{Patients}

The number of patients enrolled (between 10 March 2004 and 12 September 2005) per study center ranged from three to 32 . Some centers were unable to recruit a sufficient number of patients; therefore, four additional centers were contacted, leading to 14 investigational sites participating in this study overall. A total of 141 patients were enrolled, including 113 adult patients with a diagnosis of proven or probable IA. Of note, six of these 113 patients were diagnosed with concurrent invasive Candida infections. Twenty-eight patients were excluded from the present analysis for the following reasons: invasive Candida infection only ( $n=10$; i.e. patients without concurrent IA); possible IA $(n=6)$; children $(n=5)$; other invasive fungal infection (Microsporum canis, $n=1$; Phaeoacremonium $\mathrm{sp}$, $n=1$; Saccharomyces cerevisae, $n=1$ ); use of voriconazole for prophylaxis only $(n=2)$; non-invasive Aspergillosis $(n=1)$; and Scedosporium fusarium infection $(n=1)$.

Baseline data for patients with proven/probable IA are summarized in Table 1. Of note, neutropenia (absolute neutrophil count $<500 / \mathrm{mm}^{3}$ ) was reported in $35.3 \%$ of patients, and $91.3 \%$ were immunocompromised. The average duration of hospitalization due to IA was 9.6 days (median 3.5 days; range 0-94 days).

Treatment duration and OLAT use

Ninety-six patients were initially treated with voriconazole alone (85\%); 14 patients were switched to OLAT following an average duration of 26 days of voriconazole therapy (median 15 days; range 3-83 days) because of either insufficient response ( $n=4$ patients) or for safety reasons ( $n=10$ patients;
Table 1 Baseline data for 113 patients with acute invasive aspergillosis treated with voriconazole (intravenous or oral)

\begin{tabular}{ll}
\hline Baseline characteristics & Value \\
\hline Mean age, years (range) & $55.6(14-85)$ \\
Male, $n(\%)$ & $67(59)$ \\
Mean weight, kg ( \pm SD) & $65.4 \pm 14.4$ \\
Underlying condition, $n(\%)^{\mathrm{a}}$ & \\
Allogeneic hematopoietic stem cell transplantation & $21(18.6)$ \\
Autologous hematopoietic stem cell transplantation & $4(3.5)$ \\
Leukemia & $51(45.1)$ \\
Other hematologic malignancy & $23(20.3)$ \\
Solid tumour & $8(7.0)$ \\
Solid organ transplantation & $10(8.8)$ \\
AIDS & $2(1.8)$ \\
Pulmonary diseases & b \\
Autoimmune diseases & $8(7.0)$ \\
Liver cirrhosis & $3(2.6)$ \\
\hline
\end{tabular}

${ }^{\text {a }}$ Patients could have multiple underlying conditions

${ }^{\mathrm{b}}$ Chronic obstructive pulmonary disease $(n=7)$; tuberculosis $(n=1)$

c Wegener's granulomatosis $(n=1)$; agranulocytosis $(n=1)$; autoimmune skin disease $(n=1)$

Table 2). Four patients with a shift from voriconazole to OLAT were treated again later with voriconazole. In ten patients, OLAT was added to voriconazole after an average of 12 days (range 3-35 days), either because of insufficient response (nine patients) or due to safety reasons (one patient, who received 3 days of fluconazole following "pyrexia"; the

Table 2 Treatment characteristics for 113 adult patients with invasive aspergillosis

\begin{tabular}{|c|c|}
\hline Treatment characteristic & Value \\
\hline Voriconazole treatment duration, mean, days (range) & $63(1-183)$ \\
\hline \multicolumn{2}{|c|}{ Treatment duration in voriconazole-only patients, mean, days (range) } \\
\hline All $(n=72)$ & $56(1-183)$ \\
\hline IV only $(n=24)$ & $12(1-36)$ \\
\hline Oral only $(n=24)$ & $73(6-183)$ \\
\hline IV and oral $(n=24)$ & $83(13-183)$ \\
\hline \multicolumn{2}{|l|}{ Course of antifungal treatment, $n(\%)$} \\
\hline Voriconazole only & $72(63.7)$ \\
\hline Voriconazole $\rightarrow$ OLAT & $14(12.4)$ \\
\hline Voriconazole $\rightarrow$ combination therapy ${ }^{\mathrm{a}, \mathrm{b}}$ & $10(8.8)$ \\
\hline Combination therapy ${ }^{\mathrm{a}}$ & $13(11.5)$ \\
\hline Combination therapy $^{\mathrm{a}} \rightarrow$ OLAT $^{\mathrm{c}}$ & $4(3.5)$ \\
\hline
\end{tabular}

$I V$ intravenous, OLAT other licensed antifungal therapy

${ }^{a}$ Voriconazole plus OLAT

${ }^{b}$ Three of these patients subsequently treated with voriconazole alone

${ }^{\mathrm{c}}$ One of these patients subsequently treated with voriconazole alone 
voriconazole dose was not changed). Seventeen patients were initially treated with a combination of voriconazole and OLAT; in four of the 17 , voriconazole was subsequently stopped.

In the overall population $(N=113)$, voriconazole treatment duration ranged from 1 to 183 days (median duration 49.5 days), keeping in mind that all durations $>182$ days were recorded as 183 days. Most patients (41.6\%) received only oral formulations of voriconazole, 32 patients (28.3\%) were only treated intravenously, and $34(30.1 \%)$ received both IV and oral therapy. Among the latter, 29/34 patients $(85.3 \%)$ underwent step down from IV to oral therapy after an average IV treatment duration of 11 days (range 1-61). Oral voriconazole doses ranged from 400 to $600 \mathrm{mg} / \mathrm{d}$. Voriconazole dosages were generally in line with those recommended in prescribing guidelines [7, 8].

Most patients $(63.7 \%)$ were treated with voriconazole only (Table 2 ), with a median total treatment duration of 32 days; mean treatment durations in patients receiving only voriconazole were considerably longer in patients given oral or both IV and oral formulations than in those receiving just IV voriconazole (Table 2).

Caspofungin was the most frequent OLAT employed in this study, given to 23 of the 41 patients who received a systemic antifungal other than only voriconazole. Other antifungal agents used by smaller numbers of patients included various amphotericin B formulations $(n=12$ patients), itraconazole $(n=3)$, and fluconazole $(n=8)$. Fluconazole is not active against Aspergillus spp. but was nevertheless administered to some patients with IA only, mostly for antifungal prophylaxis or as part of salvage combination therapy.

\section{Treatment response}

Successful outcomes were observed in 50.4\% (57/113) of patients: 41 experienced a complete and 16 a partial response. Stable response was reported in 13 patients and failure in 43 patients. Among those patients who received only voriconazole, $58.3 \%$ had a successful treatment outcome (Table 3), which was higher than in patients receiving combination therapy $(48.1 \%)$ or those switched to OLAT (14.4\%).

The crude mortality rate at the end of the observational period (12 weeks) was $52.2 \%$; crude mortality at days 14 , 42 , and 84 was $14.2 \%, 27.4 \%$, and $41.6 \%$, respectively. The majority of all deaths were attributed to underlying disease and/or other causes rather than fungal infection. Only 19 deaths were attributable (wholly or in part) to invasive fungal disease, corresponding to an attributable mortality rate of $16.8 \%$ (Table 4 ). The mortality rate was lower in patients who received voriconazole monotherapy or voriconazole-based combination therapy than in those who were switched to OLAT (Table 4). Crude mortality at the end of the observational period in patients receiving exclusively IV voriconazole was $75.0 \%$ (18/24), following an average of 12 days of voriconazole treatment. The cause of death was attributable to fungal infection in three of these patients and to a combination of fungal infection and underlying disease in four others; the remaining patients died due to underlying disease $(n=10)$ or sepsis $(n=1)$.

Safety

Safety was assessed in all 119 adult IA patients, including those with possible IA $(n=6)$. Treatment-related AEs were reported for $22 / 119$ patients $(18.5 \%)$, with some patients experiencing more than one such event.

Hepatic AEs and renal toxicity were reported in 12 and four patients, respectively. Ten patients reported other AEs: confusion or hallucinations (five patients), neutropenia, dysphagia, an unspecified neurologic $\mathrm{AE}$, dysuria, gastric pain, and encephalopathy (one patient each). A shift from at least one normal laboratory test value at baseline to an abnormal value during treatment was recorded for 34 of 119 patients (28.6\%), depending on the specific laboratory test: 13 patients for alanine aminotransferase, 12 patients for aspartate aminotransferase, 14 patients for alkaline phosphatase, ten patients for bilirubin, and six patients for serum creatinine.
Table 3 Clinical response by type of treatment

OLAT other licensed antifungal therapy

\begin{tabular}{lllll}
\hline Clinical response & $\begin{array}{l}\text { All patients } \\
(n=113)\end{array}$ & $\begin{array}{l}\text { Voriconazole } \\
\text { only }(n=72)\end{array}$ & $\begin{array}{l}\text { Voriconazole switched } \\
\text { to OLAT }(n=14)\end{array}$ & $\begin{array}{l}\text { Combination } \\
\text { therapy }(n=27)\end{array}$ \\
\hline $\begin{array}{l}\text { Successful } \\
\text { response }\end{array}$ & 57 & 42 & 2 & 13 \\
$\begin{array}{c}\text { Complete } \\
\text { response }\end{array}$ & 41 & 31 & 0 & 10 \\
$\begin{array}{c}\text { Partial } \\
\text { response }\end{array}$ & 16 & 11 & 2 & 3 \\
$\begin{array}{l}\text { Unsuccessful } \\
\text { response }\end{array}$ & 56 & 30 & 12 & 14 \\
$\begin{array}{l}\text { Stable } \\
\text { Failure }\end{array}$ & 13 & 8 & 1 & 4 \\
\hline
\end{tabular}


Table 4 Death rate and causes of death by type of treatment in the 113 patients with invasive aspergillosis over the course of the observational period (12 weeks)

\begin{tabular}{lllll}
\hline & $\begin{array}{l}\text { All patients } \\
(n=113)\end{array}$ & $\begin{array}{l}\text { Voriconazole } \\
\text { only }(n=72)\end{array}$ & $\begin{array}{l}\text { Voriconazole switched } \\
\text { to OLAT }(n=14)\end{array}$ & $\begin{array}{l}\text { Combination therapy } \\
(n=27)\end{array}$ \\
\hline $\begin{array}{l}\text { Total number of deaths, } n(\%) \\
\text { Cause of death, } n(\%)\end{array}$ & $59(52.2)$ & $35(48.6)$ & $10(71.4)$ & $14(51.9)$ \\
$\begin{array}{l}\text { Underlying disease } \\
\text { Fungal infection }\end{array}$ & $34(57.6)$ & $20(57.1)$ & $6(60.0)$ & $8(57.1)$ \\
Mixed (underlying disease and fungal infection) & $8(13.6)$ & $3(8.6)$ & $1(10.0)$ & $2(14.6)$ \\
Mixed (underlying disease plus other causes $\left.{ }^{\mathrm{a}}\right)$ & $6(10.2)$ & $6(17.6)$ & $0(-)$ & $0(-)$ \\
\hline
\end{tabular}

$O L A T$ other licensed antifungal therapy

${ }^{a}$ Septic shock/septicemia $(\times 2)$, multiple organ failure, chronic respiratory failure, cerebral tumor, bowel ischemia

Three patients (all with probable/proven IA) experienced serious AEs that were considered related to voriconazole treatment, and voriconazole was discontinued in all three. The serious AEs that resulted in treatment discontinuation were as follows: (1) hallucinations on day 3 (recovered on day 4, when voriconazole was discontinued); (2) toxic nephropathy, neurotoxicity, and hepatotoxicity on day 16 in a solid organ transplant recipient with hematologic malignancy (this patient eventually died from underlying disease); and (3) confusion with encephalopathy on day 183 in a patient receiving combination therapy with voriconazole, caspofungin, and conventional amphotericin B (recovered completely).

In total, 22 of $119(18.5 \%)$ patients discontinued voriconazole treatment because of safety issues, i.e., all patients for whom an $\mathrm{AE}$ was reported. Among the remaining patients, $43(36.1 \%)$ discontinued voriconazole treatment following therapeutic failure, $53(44.5 \%)$ because the patient responded to treatment (not broken down by type of treatment), and one for "other" reasons (not specified by the investigator).

\section{Discussion}

This observational study supports the results from previous clinical trials indicating the efficacy and good tolerability of voriconazole in the treatment of IA. Investigators were not bound to adhere to a specific protocol of antifungal therapy. The results of this study thus reflect a range of available treatment options and strategies.

About half of all adult patients given voriconazole as first-line therapy for proven/probable IA in a real-life setting recorded a successful treatment outcome. Of note, more than $40 \%$ of all patients received only oral voriconazole, and an additional $25 \%$ of patients were stepped down from IV to oral voriconazole after an average of 11 days' therapy. These findings support previous suggestions that a large number of patients can be effectively treated with oral voriconazole [21]. However, current guidelines of the Infectious Diseases Society of America (IDSA) recommend initial use of parenteral therapy where feasible in patients with IA [16], reflecting the design of the original randomized clinical trial of voriconazole versus amphotericin B [13]. The majority of patients who were switched to OLAT did so because of safety concerns about voriconazole, rather than presumed lack of response. Almost three quarters of those switched to other antifungals had died by the end of the observational period, most of them from their underlying disease; this does not necessarily indicate lack of efficacy of OLAT, since it is possible that these patients had more severe underlying disease than those who continued taking voriconazole.

About $25 \%$ of all patients received voriconazole-based antifungal combination therapy at some point during the study, mostly with other mould-active agents. Mortality in these patients was similar to that observed in patients treated with voriconazole only, but clinical success rates were $10 \%$ lower, possibly reflecting more severe fungal disease. Among patients given only voriconazole, treatment durations were shorter in those administered just the IV formulation; the data suggest that those who received only IV voriconazole died earlier, before step down to an oral formulation could take place.

Overall, voriconazole was found to be well tolerated. Since all patients for whom an AE was reported discontinued the study, it is probable that AEs that did not lead to treatment discontinuation were not reported by the investigators.

Observational studies are a key complement to clinical trials in the same setting, and can verify that treatment outcomes observed under the highly controlled environment of a clinical study are reproducible in real-life situations. The pivotal clinical trial conducted by Herbrecht et al. [13] supports the use of voriconazole as the gold standard for first-line treatment of IA [14-17]. Of note, 
clinical outcomes (i.e., treatment success at 12 weeks) for proven/probable IA were almost identical between our observational study and the clinical trial $[13,22]$. The results of our study, including clinical outcomes, frequency of combination therapy, and mortality, are also similar to those from observational studies assessing the treatment of invasive fungal disease in critically ill patients and hematology patients undergoing intensive chemotherapy $[23,24]$.

On the other hand, the mortality rate of $52 \%$ in our study was much higher than the $29 \%$ in the clinical trial reported by Herbrecht et al. [13]. However, different cut-off points were used in the observational study and clinical trial (i.e., an observational period of a maximum of 182 days vs. day 84 , respectively); when extrapolating the results to day 84 , the crude mortality rate in this observational study was only $42 \%$. Mortality rates were $27 \%$ and $20 \%$ on day 42 in our observational study and the clinical trial, respectively, and $14 \%$ and $9 \%$ on day 14 . Survival was therefore somewhat lower at all time points than in the clinical trial. However, this may not come as a surprise, given that our observational study included patients with poor prognosis at baseline, who would have been excluded from enrollment in the trial. A systematic comparison of observational studies and randomized clinical trials found that mortality rates can be higher in the former, due to differences in mortality risk of the enrolled patients [25]. The higher mortality rate observed in our study may also explain why the median total treatment duration in patients remaining on voriconazole monotherapy (50 days) was lower than that reported by Herbrecht et al. (77 days) [26]. It should be noted that successful treatment response in our study was observed almost exclusively in patients receiving voriconazole monotherapy or voriconazole-based combination therapy. Treatment failed in all but two of the 14 patients switched to OLAT.

The good tolerability of voriconazole observed in our study is in line with the known safety profile of this antifungal [7, 8]. However, except for the incidence of hallucinations $(\sim 5 \%)$, the frequency of AEs observed was somewhat higher than that reported in clinical trials; this was particularly the case for abnormal liver function tests $(\sim 5 \%)$ and increased creatinine levels $(\sim 1 \%)$. This discrepancy may be due to differences in the definitions of abnormal laboratory parameters and differences in the patient populations. On the other hand, only severe or unexpected AEs were reported in our study and AEs may thus actually have been underreported. Of note, none of the laboratory abnormalities required treatment or management other than the discontinuation of voriconazole.

While observational studies are important complements to randomized clinical trials [18], they also have certain weaknesses, including selection and information bias. Our study allowed the inclusion of patients with very poor prognosis, thus complicating comparison of the results with those from clinical trials. Unfortunately, the specific type of IA (i.e., pulmonary, extrapulmonary, disseminated, or cerebral) was not recorded. Another potential limitation is the difficulty in accurately assessing clinical response in patients who received only a few days of treatment. Furthermore, the study did not use well-defined criteria for the evaluation of treatment response. Since response was assessed on a case-by-case basis by the investigator, possibly using somewhat different principles and methods of evaluation, survival may be a more meaningful outcome measure in our study than clinical response. On the other hand, the study design closely mimics clinical reality, which constitutes a considerable strength. Due to the nature of IA, which is a severe and life-threatening infection, management is based on generally accepted recommendations. Therefore, it is unlikely that participation in an observational study would have influenced the treatment of the patients enrolled. The assessment and diagnosis of IA was carried out in strict accordance with the standard criteria defined by the EORTC-MSG [20], as was also done in the pivotal clinical trial [13]. Despite the inherent shortcoming of any observational study, our data allow robust conclusions on the usage of voriconazole in a reallife setting.

This observational study confirms the findings of previous clinical trials, which demonstrated voriconazole to be an effective agent for the treatment of IA. Adverse events observed in this study were also consistent with the known safety profile of voriconazole.

Acknowledgements The authors wish to express their gratitude to the other investigators of the study group: Prof. A. Bosly, Cliniques Universitaires UCL, Godinne; Dr. G. Bries, Virga Jesse Ziekenhuis, Hasselt; Prof. P. Damas, CHU Sart-Tilman, Liège; Prof. A. Ferrant and Prof. P.- F. Laterre, Cliniques Universitaires St-Luc, Bruxelles; Dr. M. Lambrechts, AZ St Maarten, Mechelen; Dr. J. Maertens, UZ Gasthuisberg, Leuven; Dr. A. Van Hoof and Dr. J. Van Droogenbroeck, AZ St Jan, Brugge; Prof. H. Spapen, AZ VUB, Jette; Dr. A. Vergison, HUDERF, Laeken; Prof. P. Zachée, Alg Centrumziekenhuis Antwerpen, Stuivenbergh. The authors also wish to thank Dr. Vincent De Mees for his contribution to the study design and analysis, Katelyne Capiau, Pfizer, for her input as study coordinator, Annelies Decoster for the monitoring and field work, Dr. Marie-Paule Derde, Veeda Clinical Research, for the data management and statistical analysis, and Hilde Van Campenhout, Pfizer, for the overall study management.

The study was sponsored by Pfizer. Editorial support was provided by Dominik Wolf, MSc, of PAREXEL and funded by Pfizer International Operations.

Potential conflicts of interest F. Jacobs has received consultancy fees from Pfizer and other pharmaceutical companies that market antifungal agents. D. Selleslag received honoraria for public speaking from Pfizer and MSD, and research grants from Pfizer, Basilea, and MSD. M. Aoun, A. Sonet, and A. Gadisseur have no relevant conflicts of interest to declare. 
Open Access This article is distributed under the terms of the Creative Commons Attribution Noncommercial License which permits any noncommercial use, distribution, and reproduction in any medium, provided the original author(s) and source are credited.

\section{References}

1. Maschmeyer G, Haas A, Cornely OA (2007) Invasive aspergillosis: epidemiology, diagnosis and management in immunocompromised patients. Drugs 67:1567-1601

2. Enoch DA, Ludlam HA, Brown NM (2006) Invasive fungal infections: a review of epidemiology and management options. J Med Microbiol 55(Pt 7):809-818

3. Marr KA, Carter RA, Crippa F, Wald A, Corey L (2002) Epidemiology and outcome of mould infections in hematopoietic stem cell transplant recipients. Clin Infect Dis 34:909-917

4. Ohmagari N, Raad II, Hachem R, Kontoyiannis DP (2004) Invasive aspergillosis in patients with solid tumors. Cancer 101:2300-2302

5. Lin SJ, Schranz J, Teutsch SM (2001) Aspergillosis case-fatality rate: systematic review of the literature. Clin Infect Dis 32:358-366

6. Kibbler CC, Seaton S, Barnes RA, Gransden WR, Holliman RE, Johnson EM, Perry JD, Sullivan DJ, Wilson JA (2003) Management and outcome of bloodstream infections due to Candida species in England and Wales. J Hosp Infect 54:18-24

7. European Medicines Agency (EMA) Vfend: EPAR - product information. Available at URL: http://www.ema.europa.eu/docs/ en_GB/document_library/EPAR___Product_Information/human/ 000387/WC500049756.pdf. Accessed 26 September 2011

8. Pfizer Inc. (2010) Vfend (voriconazole) US prescribing information. Last revised December 2010. www.pfizer.com/files/products/ uspi_vfend.pdf. Accessed 26 September 2011

9. Alastruey-Izquierdo A, Castelli MV, Cuesta I, Zaragoza O, Monzón A, Mellado E, Rodríguez-Tudela JL (2009) In vitro activity of antifungals against Zygomycetes. Clin Microbiol Infect 15(Suppl 5):71-76

10. Walsh TJ, Pappas P, Winston DJ et al (2002) Voriconazole compared with liposomal amphotericin B for empirical antifungal therapy in patients with neutropenia and persistent fever. $\mathrm{N}$ Engl J Med 346:225-234

11. Denning DW, Ribaud P, Milpied N, Caillot D, Herbrecht R, Thiel E, Haas A, Ruhnke M, Lode H (2002) Efficacy and safety of voriconazole in the treatment of acute invasive aspergillosis. Clin Infect Dis 34:563-571

12. Perfect JR, Marr KA, Walsh TJ et al (2003) Voriconazole treatment for less-common, emerging, or refractory fungal infections. Clin Infect Dis 36:1122-1131

13. Herbrecht R, Denning DW, Patterson TF et al (2002) Voriconazole versus amphotericin $\mathrm{B}$ for primary therapy of invasive aspergillosis. N Engl J Med 347:408-415
14. Böhme A, Ruhnke M, Buchheidt D et al (2009) Treatment of invasive fungal infections in cancer patients - recommendations of the Infectious Diseases Working Party (AGIHO) of the German Society of Hematology and Oncology (DGHO). Ann Hematol 88:97-110

15. Thursky KA, Playford EG, Seymour JF, Sorrell TC, Ellis DH, Guy SD, Gilroy N, Chu J, Shaw DR (2008) Recommendations for the treatment of established fungal infections. Intern Med $\mathrm{J}$ 38:496-520

16. Walsh TJ, Anaissie EJ, Denning DW et al (2008) Infectious Diseases Society of America. Treatment of aspergillosis: clinical practice guidelines of the Infectious Diseases Society of America. Clin Infect Dis 46:327-360

17. Maertens J, Marchetti O, Herbrecht R et al (2010) European guidelines for antifungal management in leukemia and hematopoietic stem cell transplant recipients: summary of the ECIL 3-2009 Update. Bone Marrow Transplant 46:709-718

18. Black N (1999) What observational studies can offer decision makers. Horm Res 51(Suppl 1):44-49

19. Van Campenhout H, Marbaix S, Derde MP, Annemans L (2008) Voriconazole treatment of invasive aspergillosis: realworld versus health-economic model results. Clin Drug Investig 28:509-521

20. Ascioglu S, Rex JH, de Pauw B et al (2002) Defining opportunistic invasive fungal infections in immunocompromised patients with cancer and hematopoietic stem cell transplants: an international consensus. Clin Infect Dis 34:7-14

21. Leather H (2005) Economic effects of aspergillosis management in high-risk patients. Manag Care Interface 18:46-52

22. Patterson TF, Boucher HW, Herbrecht R et al (2005) Strategy of following voriconazole versus amphotericin B therapy with other licensed antifungal therapy for primary treatment of invasive aspergillosis: impact of other therapies on outcome. Clin Infect Dis 41:1448-1452

23. Slobbe L, Polinder S, Doorduijn JK, Lugtenburg PJ, el Barzouhi A, Steyerberg EW, Rijnders BJ (2008) Outcome and medical costs of patients with invasive aspergillosis and acute myelogenous leukemia-myelodysplastic syndrome treated with intensive chemotherapy: an observational study. Clin Infect Dis $47: 1507-1512$

24. Alvarez-Lerma F, Nicolás-Arfelis JM, Rodríguez-Borregán JC, Díaz-Regañón J, Sa-Borges M, García-López F, Allepuz-Palau A (2005) Clinical use and tolerability of voriconazole in the treatment of fungal infections in critically ill patients. J Chemother $17: 417-427$

25. Benson K, Hartz AJ (2000) A comparison of observational studies and randomized, controlled trials. $N$ Engl J Med 342:1878-1886

26. Herbrecht R, Natarajan-Ame S, Letscher-Bru V, Canuet M (2004) Invasive pulmonary aspergillosis. Semin Respir Crit Care Med 25:191-202 